Beate Kierey

\title{
Begleitung von Patienten mit Angststörungen in der ambulanten Pflege
}

Angst ist ein besonderes Gefühl der Seele. Sie erhöht als existenzielle Notwendigkeit die Wachsamkeit in vermeintlich oder tatsächlich gefahrvoller Situation. Angst kommt auf, wenn das «Ich-Gefühl» von einer plötzlichen und unangenehmen Situation, die sich als bedrohlich manifestiert, herausgefordert wird.

Wie erleben wir Angst? Spürbar wird, dass die innere Ruhe verloren geht sowie die allgemeine Aufmerksamkeit abnimmt und sich eher auf wenige Objekte einengt. Man kann auch sagen, dass das «Ich» im Leib versinkt und keinen Halt mehr in sich selbst findet.

Ein inneres Zittern entsteht, weiche Beine werden spürbar, der Wille und die eigene Handlungsfähigkeit sind eingeschränkt. Der Atem ist erschwert, das Herz beginnt rasch zu schlagen, Schwindel erfasst den gesamten Menschen.

\section{Angststörungen}

Eine Angststörung, die sich gegenüber gesunden Angstreaktionen durch das Fehlen akuter Gefahren und/oder Bedrohungen auszeichnet, ist dann derart verstärkt, dass sie einen Krankheitswert erreicht und sich folglich einschränkend auf die Bewältigung des Alltags auswirken kann.

Allgemein werden fünf Angststörungen unterschieden [1]: Der Begriff der Agoraphobie umfasst nicht nur Ängste vor weiten und offenen Plätzen, sondern auch vor Menschenan- sammlungen, beim Fahren in Verkehrsmitteln oder beim Reisen allgemein. Das Vermeidungsverhalten kann zu häuslicher Isolierung führen.

Menschen mit einer sozialen Phobie haben Angst, im Mittelpunkt der Öffentlichkeit zu stehen. Symptome wie Herzklopfen, Zittern, Schwitzen, der Drang zum Wasserlassen, Erröten und Übelkeit können dabei auftreten.

Menschen mit spezifischen Phobien haben die Furcht vor bestimmten Objekten oder Situationen - wie z.B. Höhenangst oder Angst vor speziellen Tieren wie Spinnen oder Hunden. Die Angst vor einem Aufenthalt in geschlossenen Räumen wird dabei als Klaustrophobie bezeichnet. Da spezifische Phobien weit verbreitet sind, wird eine Diagnose nur gestellt, wenn ein erheblicher Leidensdruck besteht.

Menschen mit Panikstörung dagegen leiden unter wiederholten Panikattacken, die unvermittelt und nicht durch eine bestimmte Situation hervorgerufen werden. Kennzeichnend für Panikstörungen sind plötzlich eintretende Angst, Herzklopfen, Schwindel, Brustschmerz, Erstickungsgefühl usw.

Bei einer generalisierten Angststörung spüren die Menschen eine allgemeine und anhaltende Angst, die nicht situationsgebunden ist. Der Mensch macht sich viele Sorgen, hegt unrealistische Befürchtungen und ist häufig angespannt und vegetativ übererregt.

Laut Rolf Heine [1] erkranken 15\% der Menschen im Laufe ihres Lebens an einer Angststörung - darunter Frauen doppelt so häufig wie Männer.
Vielfach weisen Patienten gleichzeitig mit einer Angststörung depressive Symptome auf [2].

\section{Aus der Praxis für die Praxis}

Auch in der ambulanten Pflege der Lukaspflege e.V. in Freiburg, die um die anthroposophische Pflege erweitert ist, begleitet man Menschen mit unterschiedlichen Angststörungen.

\section{Praxisbeispiel}

Eine 86-jährige Frau mit rezidivierender depressiver Störung, chronischen Schmerzstörungen mit somatischen und psychischen Faktoren, «Burning-Mouth-Syndrom» und episodisch paroxysmaler Angst wird in ihrer häuslichen Umgebung zweimal täglich von einer Fachkraft der Lukaspflege e.V. besucht. Die Patientin ist sehr selbständig und bewältigt ihren Haushalt, Einkauf usw. mithilfe einer Haushaltshilfe und einer Sozialbetreuerin. Ihre Söhne leben nicht in derselben Stadt, stehen aber in regelmässiger Verbindung zu ihr. Ehrenamtlich hilft die Patientin einmal wöchentlich in einem nahegelegenen «Kleiderladen für Bedürftige».

Bisher hat die Patientin sich selbst gut einschätzen können, wenn es zur Verstärkung einer depressiven Episode kommt. Nach Rücksprache mit ihrem Psychiater und Hausarzt erfolgte mehrmals eine stationäre Einweisung. Nach Abschluss der dortigen AkutTherapie und Stabilisierung konnte

\section{KARGER}

(C) 2017 S. Karger GmbH, Freiburg

Fax +497614520714 
die Patientin jedes Mal wieder in ihre häusliche Umgebung zurückkehren.

Wichtig ist dann die Begleitung bei und zu einem geregelten Aktivitätsrhythmus mit Ruhemomenten. Mit der Erstellung einer Aufgabenliste, die mit angenehmen und positiv besetzen Tätigkeiten gekoppelt ist, erfolgt eine positive Bestärkung in der Alltagsbewältigung.

\section{Pflegerische Gesichtspunkte}

Aufgrund der Symptome erweisen sich äussere Anwendungen wie Rhythmische Einreibungen (Teil- oder Ganzkörper), Fussbäder oder Wickel als sehr wirksam. Am Morgen spürt die Patientin «ihre Depression» stärker. Je nach Befinden wird beim Morgenbesuch durch die Fachkraft die begleitende Hilfe abgestimmt, und es erfolgen Blutdruckkontrolle und Medikationsgabe.

In den Tag zu kommen fällt schwer Hilfreich zeigen sich dabei eine rhythmische Rückenabwaschung mit Rosmarin-Aktivierungsbad von Wele$\mathrm{da}^{\circledR}$ und ein ermutigendes Gespräch unter Berücksichtigung des Tagesplans. Gegebenenfalls erfolgt eine Kontaktaufnahme mit dem behandelnden Hausarzt und Therapeuten.

Besonders belastend ist für die $\mathrm{Pa}$ tientin ein schmerzhaftes «BurningMouth-Syndrom». Durch eine sinnvolle Beschäftigung im Haushalt kann sie sich jedoch von den Begleiterscheinungen der Krankheit immer wieder ablenken.

\section{Angstattacken}

Auch treten bei der Patientin unterschiedlich starke Angstattacken auf, die mit einer flachen Atmung, Herzklopfen und Schwindelgefühlen einhergehen. Angst ist eng verknüpft mit dem menschlichen Atem - beide bedingen sich gegenseitig. Die sensible Patientin spricht in diesen Fällen sehr gut auf die Rhythmischen Einreibun-

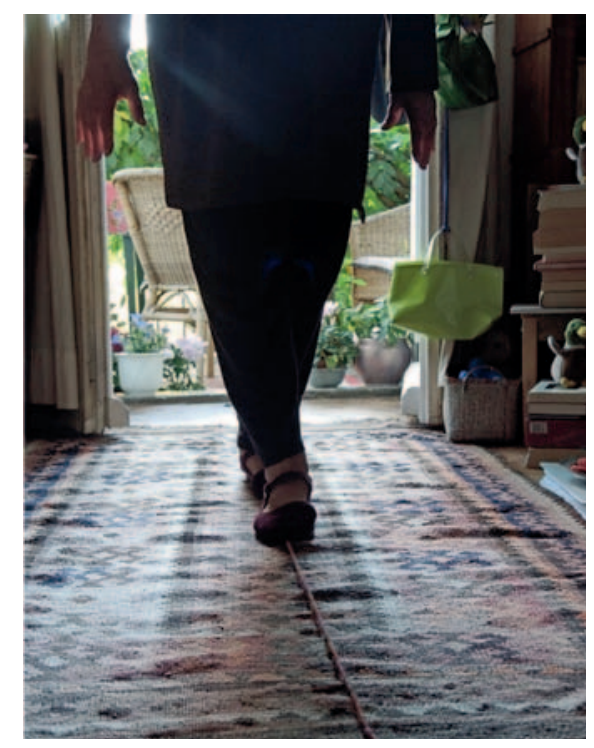

Abb. 1. Das Balancieren auf einem Wollfaden hilft, den Gleichgewichtssinn zu beleben sowie das Gefühl von Sicherheit, von Ordnung des «Ich-Gefühls» im Leib, von Klarheit und von Souveränität zu stärken (Foto: B. Kierey).

gen nach Wegman/Hauschka an. Bewährt hat sich bei ihr die Zwei-HandRückeneinreibung mit Lavendelöl. Da der Rücken als hinterer Teil des Brustbereiches das Herz, die Lunge und die Wirbelsäule umgibt, hat er einen direkten Bezug zum rhythmischen System. Die Rhythmische Einreibung des Rückens wirkt ausgleichend und harmonisierend, gleichermassen auf Herzschlag und Atmung. Zudem wirkt sie «beruhigend und lösend auf jede Art seelischer Bedrängnis, indem sie hilft, die eigene Mitte zu finden», wie es bei Monika Fingado heisst [3, S. 97].

Beim Abendbesuch führt die Fachkraft, je nach Bedarf, ein LavendelFussbad mit anschliessender Rhythmischer Fusseinreibung durch. Hier hat sich die Verwendung der «Kupfer Salbe rot» von WALA ${ }^{\circledR}$ bewährt. Warme Strümpfe und eine Wärmflasche wirken zusätzlich durchwärmend. Die häufig kalten Füsse der Patientin werden von ihr dann wieder bewusst wahrgenommen. So werden durch die äusseren Anwendungen die «unteren Sinne» sowie ihre innere "Aufrichtekraft» angesprochen und eine stabilisierende Wirkung erzielt [3, S. 71].

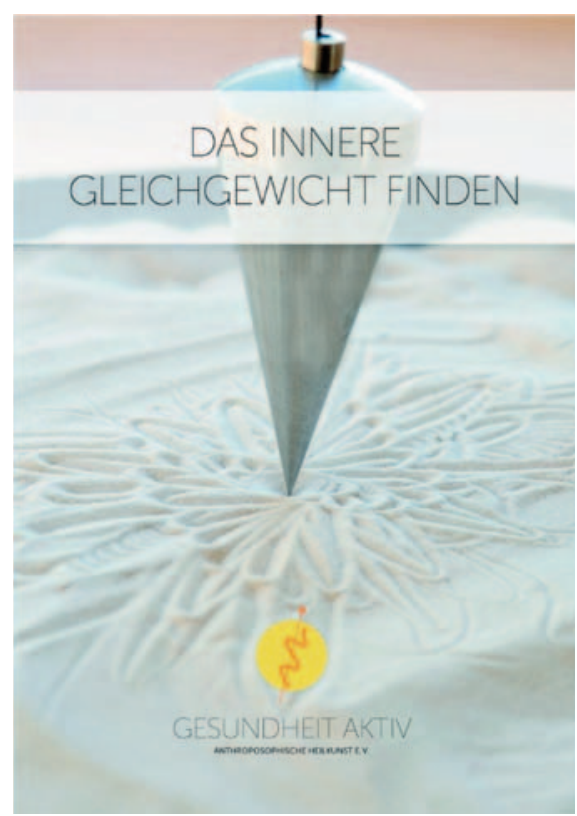

Abb. 2. Die Broschüre «Das innere Gleichgewicht finden» [4].

\section{Sicherheitsgefühl vermitteln}

Bei starken und akut empfundenen Ängsten kann die Patientin stets die Rufbereitschaft kontaktieren. Umfangreiche und zeitintensive Einsätze sind manchmal erforderlich, um sie wieder $\mathrm{zu}$ beruhigen und $\mathrm{zu}$ sich $\mathrm{zu}$ holen.

Seelenübungen zur Vertiefung der Atmung - Begleitung durch den Alltag

Es gilt, neben ermutigenden Gesprächen, auch gemeinsame Übungen in die Besuche einfliessen zu lassen, um einer erneut auftretenden Episode vorzubeugen.

Die Patientin benötigt Ablenkungen und Anregungen von aussen, vor allem Achtsamkeits- und Willensübungen. Bei diesen «Seelenübungen» geht es zunächst um ein Üben der unteren Sinne, um dem «Ich» wieder Halt und Sicherheit im Leib zu geben. Hier finden Gleichgewichtsübungen wie z.B. das Balancieren auf einem Wollfaden Anwendung (Abb. 1). Diese Übung wird bekanntlich in einer stationären Einrichtung mit einer 
Dachlatte angeleitet - im ambulanten Bereich kann dazu ein bunter Faden ausgerollt werden.

Weitere Beispiele sind eine Pflanzenbetrachtung anhand des leuchtend gelben Johanniskrauts im Sommer, das Ertasten z.B. einer herbstlichen Kastanie mit geschlossenen Augen, das Erfühlen eines Balls im Fussbad oder das tägliche Notieren positiver

\section{Literatur}

1 Heine R (Hrsg): Anthroposophische Pflegepraxis. Grundlagen und Anregungen für alltägliches Handeln. Kapitel 2.3. Angststörungen. Berlin, Salumed, 2015, p 392.
Gedanken. Ebenfalls hilfreich ist das langsame Abschreiben eines jahreszeitlich passenden Gedichts in ein sogenanntes «Sonnenbuch». Auch diese Seelenübungen werden von den Fachkräften angeregt und von der Patientin als sehr hilfreich erlebt.

Die angeführten Übungen und Massnahmen dienen dazu, die "IchPräsenz» und die innere Klarheit der
Patientin zu stärken, und verhelfen ihr bis zum heutigen Tag, selbstbestimmt in der eigenen Wohnung zu leben. Wertvolle Anregungen für Fachkräfte, pflegende Angehörige und Interessierte vermittelt dazu auch die Broschüre «Das innere Gleichgewicht finden» (Abb. 2) [4].

2 Hamre H, et al: Anthroposophische Therapie bei Angststörungen: eine zweijährige prospektive Kohortenstudie in der routinemäßigen ambulanten Krankenversorgung. Der Merkurstab 2010;2:150-161.

3 Fingado M: Rhythmische Einreibungen. Handbuch aus der Ita Wegman Klinik, ed 4. Dornach, Natura, 2012.
4 Adams K, Rißmann W, Roknic M: Das innere Gleichgewicht finden. Seelenübungen für Achtsamkeit, Herzenskultur und Willensstärkung. Gesundheit aktiv. Anthroposophische Heilkunst e.V., Juli 2015. 